\title{
EXPERIMENTAL OSTEOARTHRITIS INDUCED BY SELECTIVE MYECTOMY AND TENDOTOMY
}

\author{
CHRISTIANE L. ARSEVER and GILES G. BOLE
}

\begin{abstract}
A new model of osteoarthritis has been developed that utilizes an extraarticular surgical technique to alter gait and weight-bearing capabilities in the hind limbs of experimental animals. Guinea pigs, averaging $650 \mathrm{gm}$ in weight, were subjected to unilateral resection of a segment of the gluteal muscles at the sacral origin, to section of the infrapatellar ligament, or to both procedures. Progressive changes consistent with osteoarthritis developed over 10-24 weeks as judged by gross, radiologic, and microscopic findings. In addition, an increase in mean cartilage water content and ${ }^{35} \mathrm{SO}_{4}{ }^{-2}$ incorporation into proteoglycans by 10 and 14 weeks, respectively, were consistent with the chemical changes observed in early osteoarthritis. Abnormalities developed not only in the operated hind limb, but in the contralateral, unoperated hind limb at a slightly later time. Thus, this animal model provided the opportunity to evaluate a mild gait abnormality and other biomechanical influences on the evolution of osteoarthritis which is uninfluenced by any intraarticular joint trauma.
\end{abstract}

From the Division of Rheumatology, Rackham Arthritis Research Unit, the University of Michigan Medical Center, Ann Arbor, Michigan 48109-0010.

Supported in part by grants AM-20557, AM-07080, and AM-10531 from the National Institutes of Health.

Christiane L. Arsever, MD: Rheumatology Fellow, Division of Rheumatology and Postdoctoral Fellow of the Arthritis Foundation; Giles G. Bole, MD: Professor of Internal Medicine and Chief, Division of Rheumatology, Rackham Arthritis Research Unit.

Address reprint requests to Giles G. Bole, MD, University of Michigan Medical Center, Department of Internal Medicine, Division of Rheumatology, Rackham Arthritis Research Unit, Box 018, Ann Arbor, MI 48109-0010.

Submitted for publication December 27, 1984; accepted in revised form July 12,1985 .
Several animal models have been developed to study the pathologic and biochemical changes seen in osteoarthritis (OA) $(1,2)$. These animal models, among others, have included the sectioning of the anterior cruciate ligament in dogs and partial meniscectomy in rabbits (3-5). Guinea pigs have also been subjected to sectioning of the anterior cruciate ligament and medial collateral ligament (6). Finally, immobilization of knee joints of rats and rabbits has been used in other experimental models of OA (7-9).

Most models have relied primarily on an intraarticular surgical procedure to produce joint instability and to induce changes compatible with $\mathrm{OA}$. Thus, a postoperative inflammatory response may contribute to some of the early changes associated with the observed osteoarthritic lesions. In addition, contralateral joints have often been used as "controls," however, some studies now indicate that these joints are abnormal and cannot be used as a baseline for changes found in operated joints (10).

The current study describes a new experimental model of $\mathrm{OA}$ that utilizes an extraarticular surgical technique. This model offers the opportunity to examine early $\mathrm{OA}$ events uninfluenced by intraarticular postoperative inflammation or other local joint trauma. It has also been used to examine the postoperative biomechanical effect on the unoperated contralateral joints in the hind limbs of the same animals.

Finally, the cost of maintaining large animals for investigative studies has become substantial. It is cost-effective to be able to use small animals as experimental models, if the induced lesion reproduces the desired pathology and provides a sufficient amount of tissue for study. The proposed model fulfills all of these criteria. 


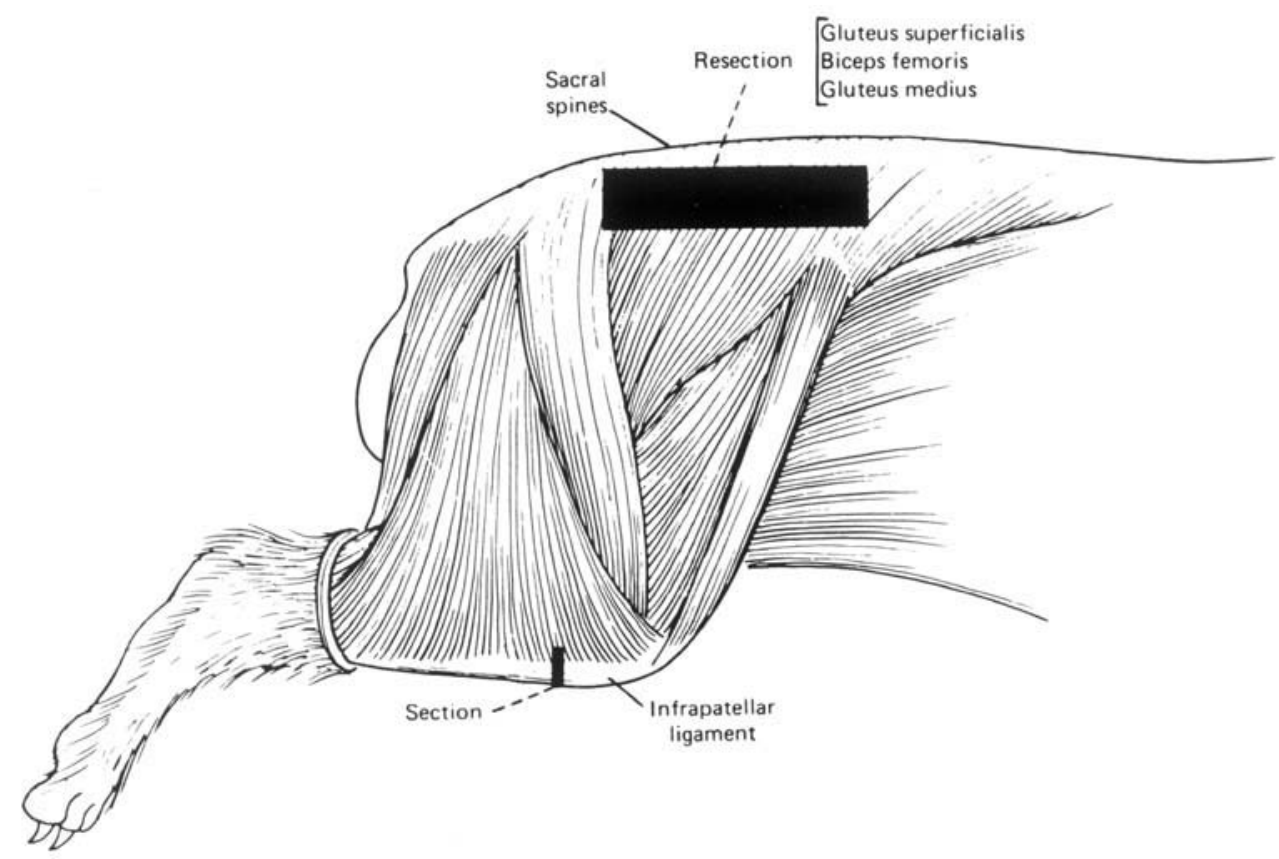

Figure 1. Illustration of a guinea pig's right hind limb. The surgical procedures involve the resection of a $0.5-1.0-\mathrm{cm} \times 2.5-3.0-\mathrm{cm}$ segment of the right gluteus superficialis', biceps femoris, and gluteus medius at their sacral origin and/or section of the right infrapatellar ligament.

\section{MATERIALS AND METHODS}

Experimental animals and surgical procedure. Fortyeight Hartley strain guinea pigs (Charles River Breeding Laboratory, Wilmington, MA), averaging $650 \mathrm{gm}$ in weight, were used in this study. Twenty-six animals had operations, 7 animals were sham-operated, and 15 were unoperated controls. The surgical procedure involved the resection of a $0.5-1.0-\mathrm{cm}$ wide $\times 2.5-3-\mathrm{cm}$ long segment of the right gluteus superficialis, biceps femoris, and gluteus medius at their sacral origins. A second procedure involved the section of the right infrapatellar ligament (Figure 1). The capsule or proximal supporting structures of the hip and knee joints were not invaded at any point. Of the 26 operated animals, 10 had both surgical procedures, 8 had only the hip procedure, and the other 8 had only the knee procedure. The sham operation involved only a skin incision at the sacral region and knee. All operated and sham-operated animals were killed at $4,8,12,16,20$, or 24 weeks postoperatively. Unoperated controls were also maintained for 4-24 weeks and killed in parallel with a matched operated animal. All animals received a supplement of $100 \mathrm{mg}$ of vitamin $C$ in their drinking water each day. Adrenal glands were removed when the animals were killed and then were assayed for vitamin $C$ content using the method of Zannoni et al (11).

Gait analysis. Gait analysis was performed on all animals by painting their hind paws with nontoxic red finger paint and walking the animals down a wooden track 9-cm wide $\times 120-\mathrm{cm}$ long lined with Whatman $3 \mathrm{MM}$ paper. The length of the stride on both sides of the midpoint of the footprints was then measured in centimeters. The average of 3 stride lengths on the right and left was taken as representative for each animal. All animals underwent gait analyses preoperatively, 2 weeks postoperatively, and thereafter, every 4 weeks until killed. The measurements were averaged and subjected to statistical analysis by Student's $t$-test.

Radiologic evaluation. Roentgenograms of the hind limbs were obtained on all animals preoperatively and immediately before they were killed. All roentgenograms were "blindly" assessed by 2 observers for joint space narrowing, subchondral sclerosis, subchondral cysts, and osteophytes.

The radiologic findings were graded blindly on a scale of $3+$ to $0+: 3+=$ a normal hip joint, $2+=$ the presence of $<50 \%$ joint space narrowing, $1+=$ the presence of $>50 \%$ joint space narrowing, mild subchondral sclerosis, and subchondral cysts, and $0+=$ complete joint space loss, severe subchondral sclerosis, subchondral cysts, and osteophytes. Osteophytes were present only when roentgenographic abnormalities were most severe.

Gross pathology and histologic studies. Hip and knee joints were exposed and inspected for cartilage thinning, surface erosions, pits, and osteophyte formation. Photographs of all joints (35- $\mathrm{mm}$ color slides) were also prepared in order to document the observed changes.

Joint specimens including articular cartilage (hip, knee, patella) were divided into equal groups for histologic study or biochemical analysis. Comparable numbers of operated and unoperated joints were placed in each group. Samples that were to undergo histologic examination were 
fixed in neutral buffered formalin and decalcified in $5 \%$ formic acid. Paraffin sections $(5 \mu)$ were stained with azure $\mathrm{A}$ and Safranin $O$ to assess metachromasia, and hematoxylin and eosin-stained to assess general histology. Histologic sections were graded blindly according to the following scale: metachromasia was graded on a scale of $3+$ (representing normal metachromasia) to 0 (representing severe loss of staining). Extracellular cartilage matrix integrity as a measure of surface fibrillation was graded from $3+$ (showing a smooth cartilage surface and intact matrix structure) to 0 (where the cartilage showed overt fibrillation). Cellularity was assessed from 3+ (representing a normal number of cells, as determined in unoperated controls) to 0 (representing a marked increase, $>50 \%$, in chondrocyte cellularity). Finally, the average articular cartilage thickness for the femoral head was determined at 2 sites, 1 near the fovea and the other at the margin, by 2 separate measurements of each specimen using an ocular micrometer. All histologic specimens were read and evaluated blindly by 2 observers.

Cartilage water content. Articular cartilage samples were removed by sharp dissection under a dissecting microscope. The samples were then placed on a watch glass in a moisture chamber (phosphate buffered saline [PBS]moistened filter paper) and allowed to equilibrate for 2 hours in the saturated environment. The samples were then rapidly weighed on a Sartorius microbalance $( \pm 30 \mu \mathrm{g})$ and dried in vacuo over $\mathrm{P}_{2} \mathrm{O}_{5}$ for 72 hours to constant dry weight at room temperature. The water content was then calculated.

Radioisotope incorporation studies. All animals were injected intraperitoneally with $200 \mu \mathrm{Ci}$ of carrier-free ${ }^{35} \mathrm{SO}_{4}{ }^{-2}$ (ICN Pharmaceuticals, Inc., Irvine, CA) 24 hours before they were killed. Each animal was placed in a metabolic cage so that urinary and fecal excretion of ${ }^{35} \mathrm{SO}_{4}{ }^{-2}$ could be determined. The cartilage from the joints was then carefully removed by sharp dissection and homogenized in a Brinkmann PT-10 polytron. The tissue proteoglycans were extracted sequentially with an associative solvent of $0.15 \mathrm{M}$ sodium acetate, $\mathrm{pH}$ 6.8, containing protease inhibitors 6aminohexanoic acid $(0.1 M)$, EDTA $(0.01 M)$, and benzamidine hydrochloride $(0.005 M)(12)$ for 16 hours at $4^{\circ} \mathrm{C}$ and the supernatant harvested by centrifugation at $100,000 \mathrm{~g}$ for 15 minutes. The residue was then treated for 24 hours at $4^{\circ} \mathrm{C}$ with a dissociative solvent of $4 M$ guanidine hydrochloride in $0.05 M$ sodium acetate, $\mathrm{pH} 5.8$, containing protease inhibitors EDTA $(0.01 M)$, 6-aminohexanoic acid $(0.1 M)$, and benzamidine hydrochloride $(0.005 M)$.

The supernatant was removed after centrifugation at $38,000 \mathrm{~g}$ for 20 minutes (13). The extracts were then dialyzed through a microdialysis system (Bethesda Research Laboratories, Gaithersburg, MD) against PBS for 24 hours using a $6,000-8,000$ molecular weight membrane and concentrated using polyethylene glycol $35 \%$ (weight/volume). Aliquots from the nondialyzable fractions of the extracts were then used to determine radioactivity by liquid scintillation counting in a Packard Model 460C spectrometer. Relative specific activity was determined for each cartilage specimen by measuring the uronic acid content (disintegrations per minute $/ \mu$ mole uronic acid) by a modified carbazole procedure (14).

\section{RESULTS}

Gait. Gross inspection of the guinea pigs' gait at 2 weeks postoperatively demonstrated that all operated animals had a limp in the right hind limb. This limp had essentially resolved by 4 weeks postoperatively. Results were similar for operated animals that underwent the hip procedure only, the knee procedure only, or both procedures.

Analysis of the measured strides (Figure 2) showed that both operated animals and control (unoperated and sham-operated) animals tended to have a longer stride during the first 8 weeks of observation, but that the stride decreased as they became older. This change correlated with somatic growth since the average body weight increased from $650 \mathrm{gm}$ to $950 \mathrm{gm}$ by 8 weeks postoperatively. Control animals and sham-operated animals had similar body weight increases during this time. As all animals became older, with an average weight of $1,050 \mathrm{gm}$ by 16 weeks, operated animals tended to have longer strides in both hind limbs compared with control animals. The difference was significant at $P<0.01$ on the right operated

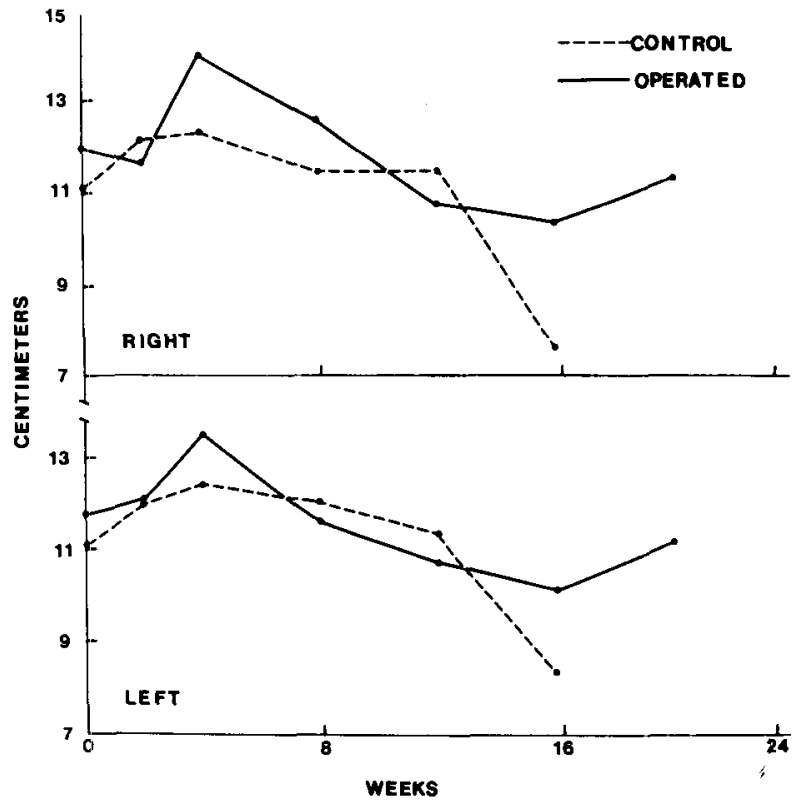

Figure 2. Gait analysis of guinea pigs with initial body weights of $600-700 \mathrm{gm}$ from 0 weeks (preoperatively) to 20 weeks postoperatively. The difference between the mean values was significant at $P<0.01$ for the right operated side at 16 weeks postoperatively compared with controls. At other time points, the difference between the means was not significant and the SEM was $\pm 0.50 \mathrm{~cm}$. 
side at 16 weeks postoperatively, compared with controls (Figure 2). At other times, the difference between the means was not significant, although there was clear evidence of a difference in the mean measured stride of both hind limbs when the control and the operated animals were compared (Figure 2).

Gross pathologic observations. All operative procedures had similar results; therefore, the operated animals were studied as one group. Gross inspection showed that in control animals the normal cartilage was white, smooth, and glistening both in the hips and knees. Operated animals, especially those animals killed after 20 weeks postoperatively, were found to have progressive articular erosive changes consistent with findings in OA. Femoral heads developed thinned and roughened surfaces in all animals; there were several areas of erosions and pits in approximately $50 \%$ of the animals by $20-24$ weeks postoperatively. Femoral condyles became slightly eroded by 12-16 weeks postoperatively and moderately eroded in all animals by $20-24$ weeks postoperatively. Tibial plateaus developed slight roughening of the cartilage in the central aspect of the medial tibial plateau by 8 weeks postoperatively in half the animals; however, this progressed to severe thinning and irregularity with mild involvement of the central aspect of the lateral tibial plateau in all animals by $20-24$ weeks postoperatively.

Patellar cartilage was found to be generally unaffected even by 24 weeks postoperatively in most animals. Osteophyte formation was not prominent, but early tibial spurs were seen in $20-30 \%$ of the knees of the operated hind limbs of the oldest experimental animals. Figure 3 shows a normal femoral head and the femoral head of an operated animal at 24 weeks postoperatively. The cartilage on the right femoral head is roughened and thinned, with several areas of surface erosions and pits. Figure 4 shows a normal tibial plateau and the tibial plateau of an operated animal at 20 weeks postoperatively. There is a knife artifact on the left side, but the medial and lateral compartments show fibrillation and roughening of cartilage with substantial loss at the margins of the joint. The severity of the changes was greater on the right, operated side; however, similar changes were also seen on the contralateral left hind limb joints.

Radiologic findings. It was found that only the hip joints could be satisfactorily evaluated by roentgenographic techniques. Evaluation of the knee joint was often compromised by positioning of the animal, and results were not reproducible on serial radiographs.

Figure 5 shows roentgenograms taken pre-

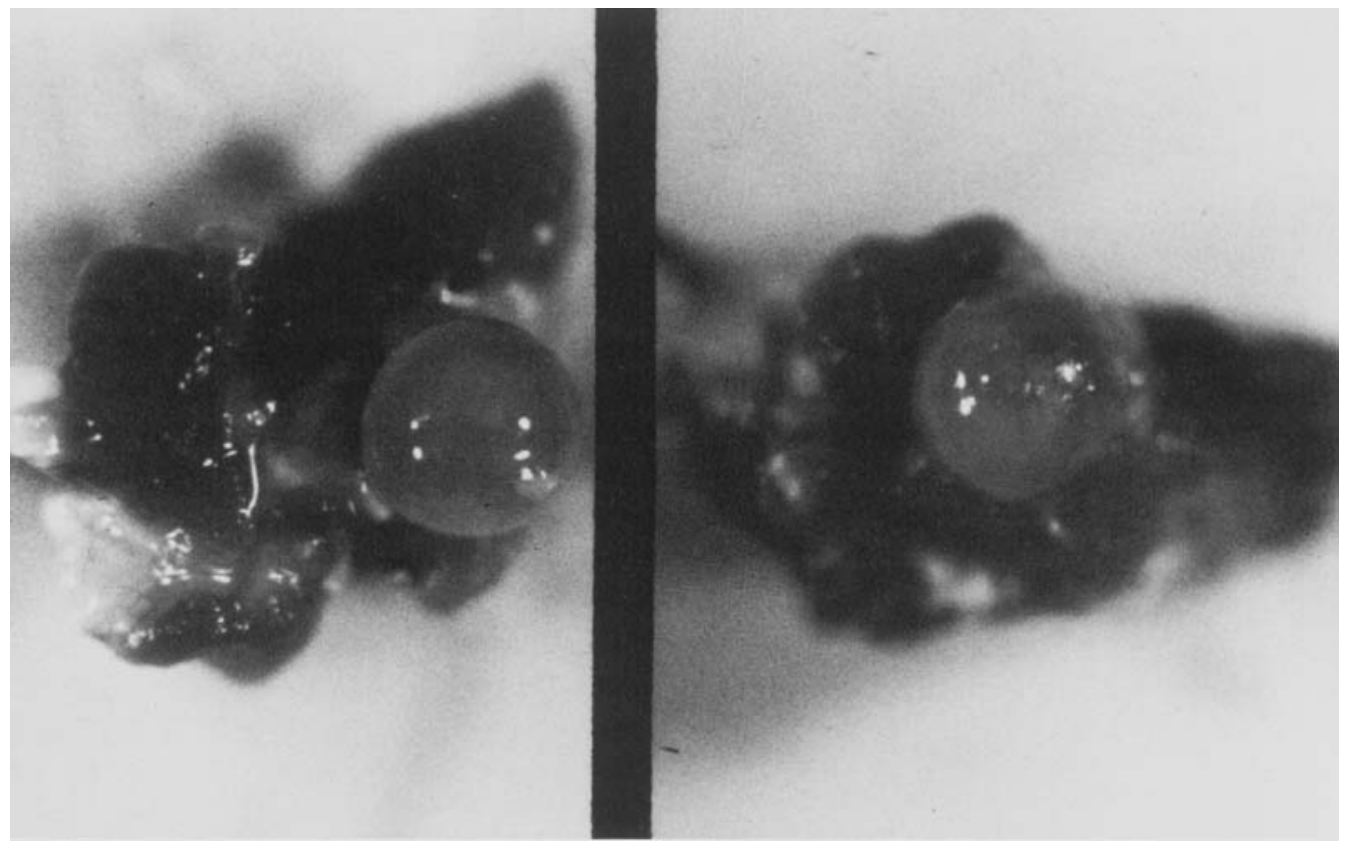

Figure 3. Left, Normal femoral head. Note the smooth and glistening cartilage. Right, Femoral head from an operated animal at 24 weeks postoperatively. The cartilage is rough, with areas of surface erosions and pits. 


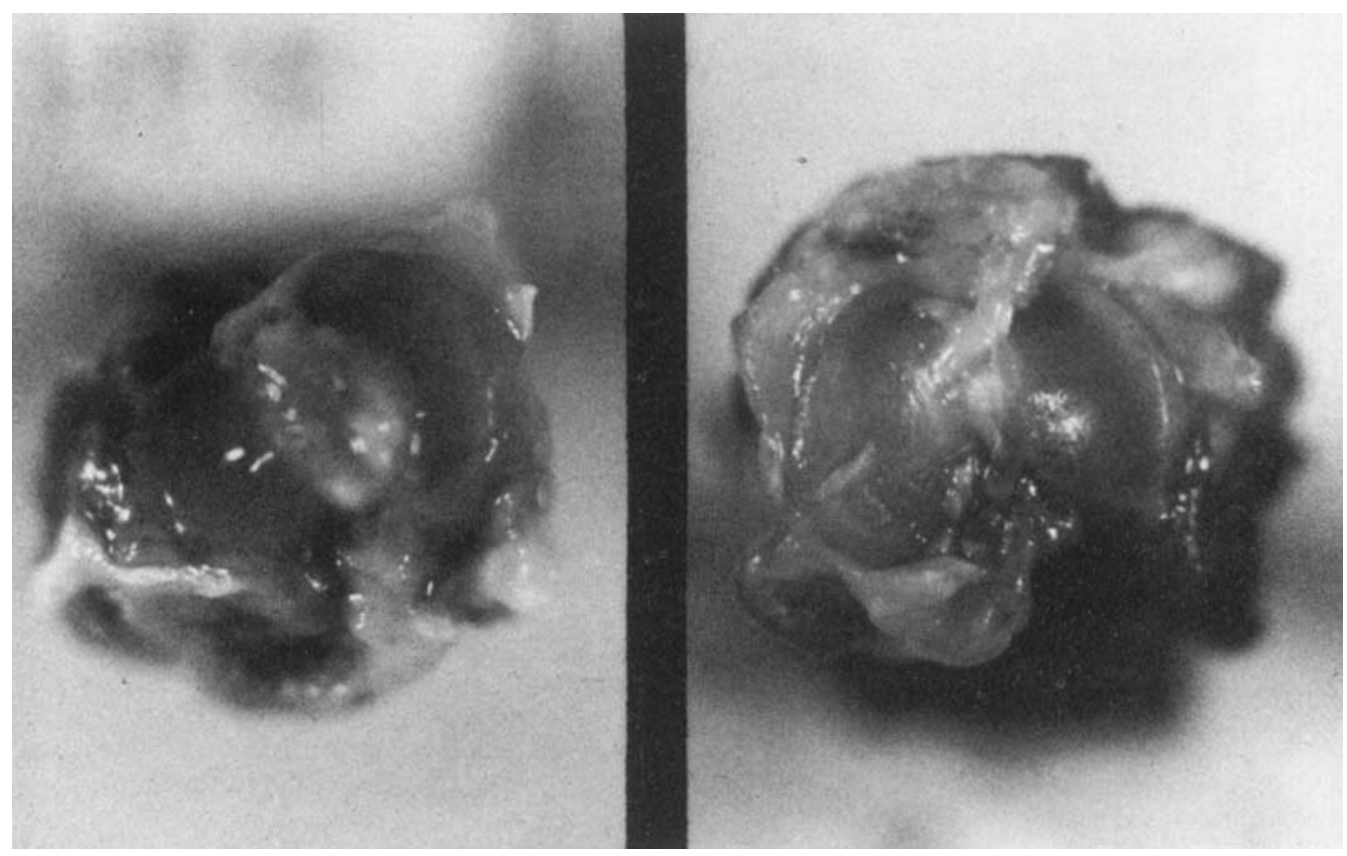

Figure 4. Left, Normal tibial plateau. Right, Tibial plateau from a 20 -week-old operated animal. Note the roughened and worn-down margins of the articular cartilage. A knife-cut artifact can be seen on the left aspect of the plateau.

operatively and 14 weeks postoperatively, immediately prior to killing, in an animal that had both gluteal myectomy and infrapatellar tendotomy. As can be seen, in the preoperative view the hip joint space can be easily defined. However, in the view taken just prior to killing, there is significant hip joint space narrowing and moderate subchondral sclerosis. Note the displaced patella, which is the result of the section of the infrapatellar ligament, on the roentgenogram taken prior to killing. Of the 22 roentgenograms from operated animals, 13 had $2+$ changes. Two animals, 1 12 weeks and the other 14 weeks postoperatively, developed $0+$ and $1+$ changes, respectively. Roentgenograms were most helpful in diagnosing $>50 \%$ joint space narrowing, subchondral sclerosis, and/or cysts in the oldest animals.

Histologic studies. The pathologic changes that developed progressively in decalcified specimens of the femoral head 8-24 weeks postoperatively (compared with controls) are listed in Table 1. Individual specimens were studied blindly and mean scores derived from analysis of 15 samples taken at each time period. Sham-operated animals and unoperated controls were grouped together since the histologic findings were the same in both groups. No evidence of postoperative inflammation was seen in any of the histologic sections of joint tissue.

Metachromasia of the femoral head cartilage, evaluated by azure A and Safranin $\mathrm{O}$ staining, demonstrated loss of staining in the operated animals as they became older. Changes in metachromatic staining of the femoral condyles and tibial plateaus were primarily seen at the superficial layers. Animals demonstrated the most prominent loss of staining at 24 weeks postoperatively.

In the femoral head, the average cartilage thickness was derived from 2 separate measurements, one taken near the fovea and the other taken at the margin of the femoral head, from 20 samples each. The cartilage became progressively thinner both at the fovea and at the margin in operated animals compared with control animals, decreasing from a mean \pm SEM of $181 \pm 8 \mu$ in controls, to $146 \pm 8 \mu$ in the oldest operated animals. The measurements of the femoral head cartilage were found to be very reproducible since the fovea was used as a marker for comparison between tissue sections and samples. On the other hand, thickness was difficult to measure in the femoral condyles and tibial plateaus due to variation in tissue 

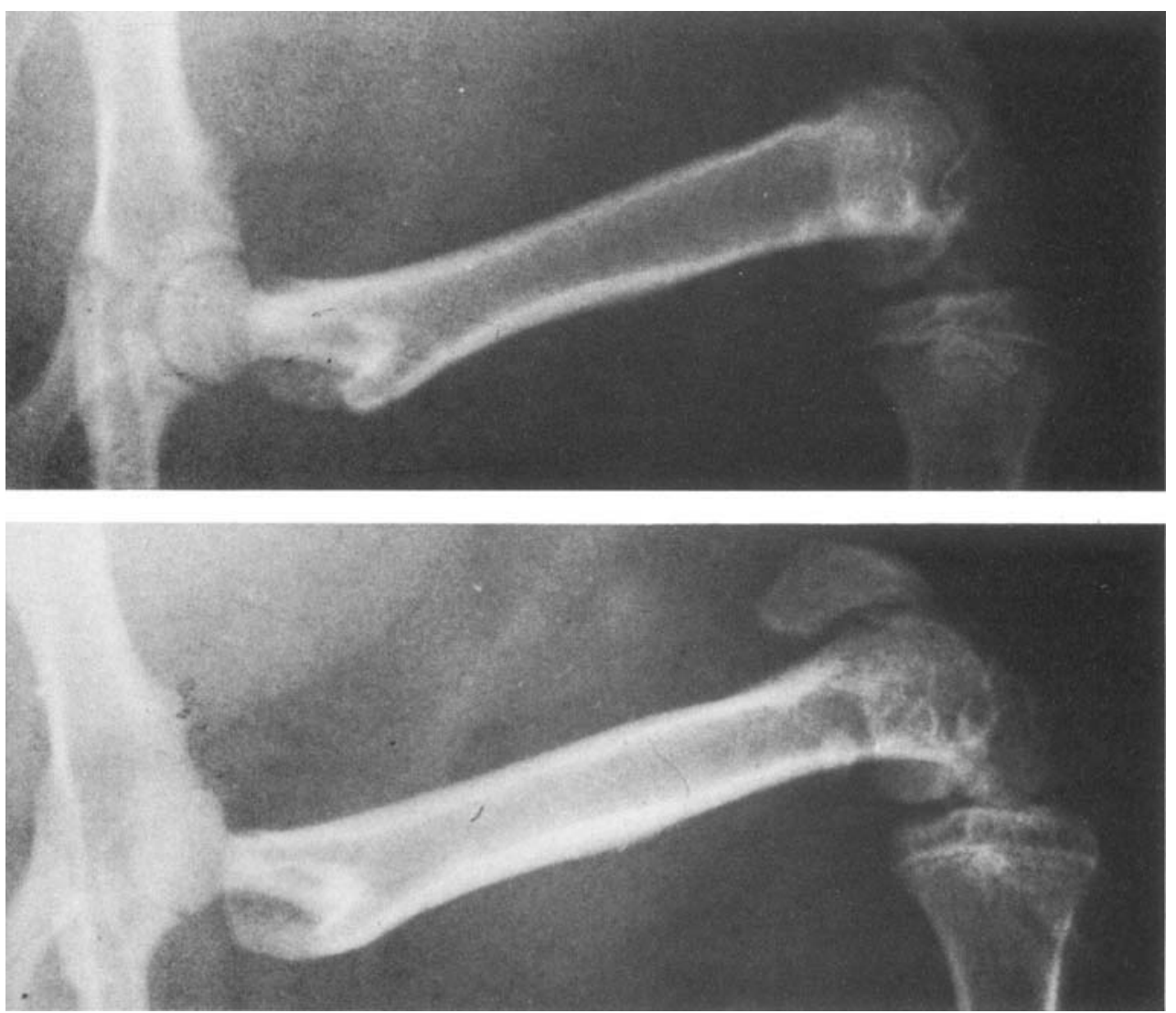

Figure 5. Top, Preoperative baseline roentgenogram. Note the width of the hip joint space. Bottom, Roentgenogram of an operated animal at 14 weeks postoperatively, immediately prior to killing. Note the significant hip joint space narrowing and subchondral sclerosis. Changes in the knee joint were difficult to evaluate due to problems in the positioning of the animals at the time of exposure of the roentgenogram.

orientation at the time of preparation of the sections; therefore, those results are not reported here.

Change in matrix integrity as a measure of surface fibrillation was minimal in the femoral head and femoral condyles. However, the tibial plateaus demonstrated surface fibrillation that progressed until 20-24-week postoperative animals showed severe fibrillation in the medial aspect of each of the tibial plateaus. Changes seen in the lateral tibial plateau were always milder compared with those seen in the medial plateau.

Figure 6 shows the rather marked fibrillation observed in Safranin O-stained section of the right medial tibial plateau of an operated animal 24 weeks postoperatively. Moderate loss of metachromatic staining can be seen at the superficial cartilage layers.

Increased cellularity of the deepest cartilage layer of the femoral head was observed, especially in the oldest operated animals. However, cellularity was within normal limits for the femoral condyles and tibial plateaus of even the oldest operated animals.

The patellar cartilage was found to be essentially normal in all aspects regardless of the age of the animals.

Vitamin $\mathbf{C}$ content. The adrenal gland content of vitamin $C$ for the operated animals was $91.2 \pm 7.4$ mg\% (mean \pm SEM) and for the control animals (unoperated and sham-operated) it was $80.5 \pm 10.9$ $\mathrm{mg} \%$. These values are well within the average range for vitamin $\mathrm{C}$ concentration in adrenal glands of guinea pigs receiving a normal diet, reported by Zannoni et al (11). Thus, the OA changes observed in this model cannot be attributed to abnormalities in vitamin $\mathrm{C}$ levels (6).

Cartilage water content. The mean percent cartilage water content for hip, knee, and patella speci- 
Table 1. Histologic evaluation of femoral head specimens, comparing differences in metachromasia and cartilage thickness, integrity, and cellularity between experimental and control animals*

\begin{tabular}{|c|c|c|c|c|}
\hline & \multirow{2}{*}{$\begin{array}{l}\text { Control } \\
\text { animals }\end{array}$} & \multicolumn{3}{|c|}{$\begin{array}{l}\text { Experimental animals } \\
\text { (weeks postoperative) }\end{array}$} \\
\hline & & 8 & 16 & 24 \\
\hline $\begin{array}{l}\text { Metachromasia } \\
\text { Osteoarthritis } \\
\text { parameters }\end{array}$ & $2.9 \pm 0.06$ & $2.8 \pm 0.15$ & $2.8 \pm 0.09$ & $2.2 \pm 0.11$ \\
\hline $\begin{array}{l}\text { Thickness } \dagger \\
\text { Integrity } \\
\text { Cellularity }\end{array}$ & $\begin{array}{l}181 \pm 8 \\
2.9 \pm 0.05 \\
2.9 \pm 0.07\end{array}$ & $\begin{array}{l}169 \pm 8 \\
2.6 \pm 0.18 \\
2.7 \pm 0.12\end{array}$ & $\begin{array}{l}163 \pm 6 \\
2.6 \pm 0.18 \\
2.6 \pm 0.18\end{array}$ & $\begin{array}{l}146 \pm 8 \\
2.5 \pm 0.22 \\
2.0 \pm 0.01\end{array}$ \\
\hline
\end{tabular}

* Values expressed as mean \pm SEM. Scale: $3+=$ normal; $2+=$ slight changes; $1+=$ moderate changes; $0=$ severe changes.

$\uparrow$ Thickness in microns as measured by a calibrated ocular micrometer. Sites evaluated include the fovea and margins.

mens 4-10 weeks and 12-16 weeks after surgery compared with control cartilage samples is shown in Table 2. Results are the average from 2-3 specimens taken from control and experimental joints at each time period. Results from 13 unoperated controls and 7 sham-operated animals have been combined as a single control value at each time point. The average yield for cartilage wet weight determinations was 8 $\mathrm{mg}, 16 \mathrm{mg}$, and $4 \mathrm{mg}$ for femoral head, knee, and patella, respectively.

The mean cartilage water content in control joints was similar in the hips and knees, but higher in the patella. On the operated right side, the mean water content was higher in the hip and knee at 4-10 weeks postoperatively than that of controls, but returned to control levels after 12-16 weeks postoperatively. The patella water content from the operated side of the animals was slightly increased over control values at both postoperative time periods.

On the unoperated left side of the animals that underwent surgery, the mean water content was lower than that in controls in all hind limb joints at 4-10 weeks postoperatively. The decrease in cartilage water content was consistent in all joints and reproducible. This suggests that the biomechanical effects of the surgical procedure were being expressed on both the operated and unoperated hind limbs. The exact mechanism for this decrease in water content has yet to be determined, but data from the ${ }^{35} \mathrm{SO}_{4}{ }^{-2}$ incorporation studies discussed later in this paper suggest that

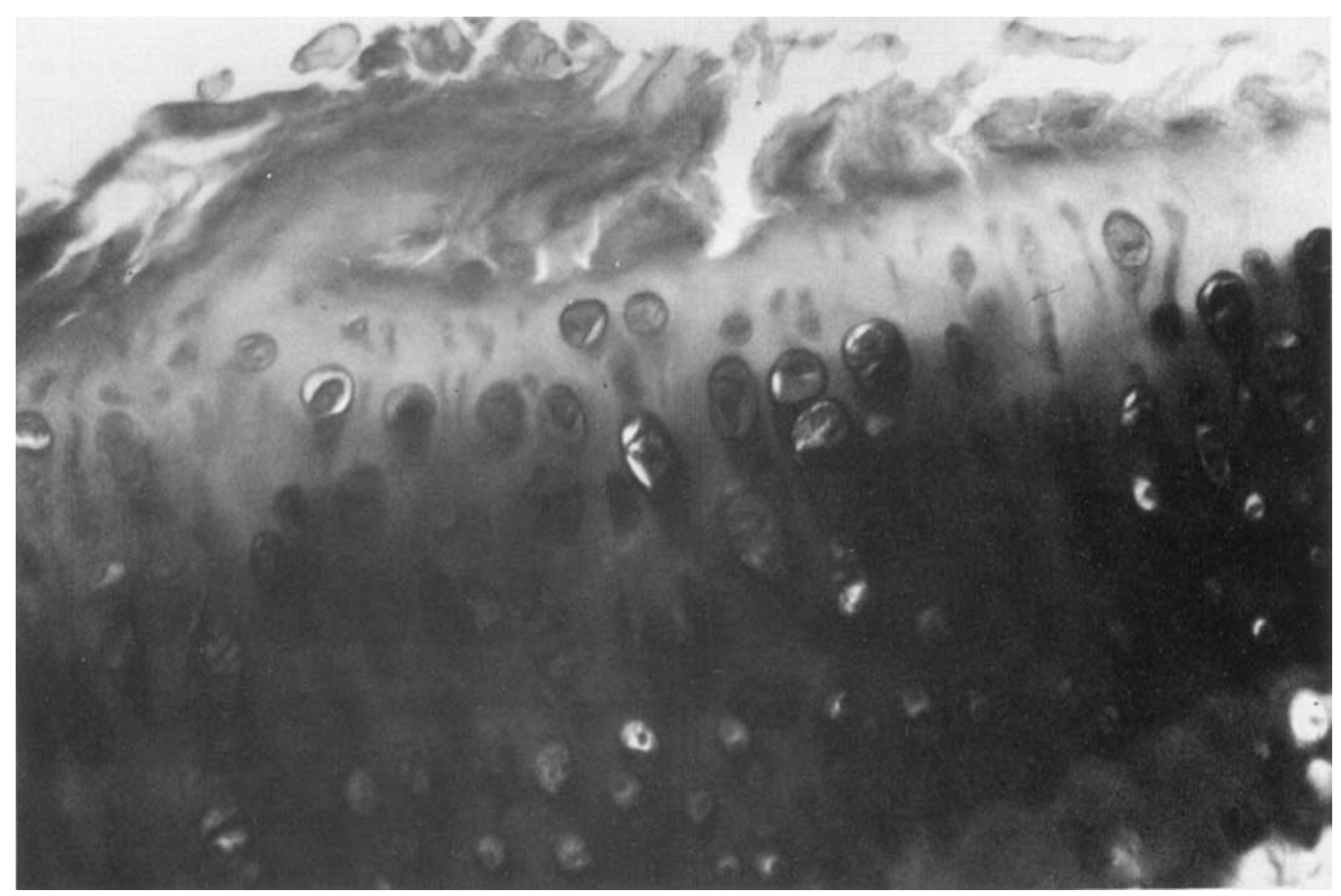

Figure 6. Safranin O-stained section $(5 \mu)$ of cartilage from the medial aspect of the right tibial plateau of an operated animal at 24 weeks postoperatively. Note overt fibrillation on the surface (top) and mild loss of metachromatic staining (original magnification $\times 400$ ). 
Table 2. Comparison of the mean cartilage water content of the femoral head, knee, and patella in specimens taken from control and experimental animals 4-10 weeks and 12-16 weeks after surgery*

\begin{tabular}{lrccc}
\hline & & & \multicolumn{2}{c}{ Experimental animals } \\
\cline { 3 - 5 } & Weeks & $\begin{array}{c}\text { Control } \\
\text { animals }\end{array}$ & $\begin{array}{c}\text { Unoperated } \\
\text { left }\end{array}$ & $\begin{array}{c}\text { Operated } \\
\text { right }\end{array}$ \\
\hline Femoral head & $4-10$ & $63 \pm 2.3$ & $57 \pm 5.7$ & $67 \pm 3.6$ \\
Knee $\dagger$ & $12-16$ & $65 \pm 1.2$ & $66 \pm 3.4$ & $65 \pm 2.1$ \\
& $4-10$ & $66 \pm 1.4$ & $61 \pm 0.1$ & $75 \pm 9.3$ \\
Patella & $12-16$ & $66 \pm 0.1$ & $70 \pm 2.3$ & $66 \pm 1.7$ \\
& $4-10$ & $76 \pm 1.9$ & $71 \pm 0.5$ & $78 \pm 4.2$ \\
& $12-16$ & $78 \pm 3.2$ & $81 \pm 7.7$ & $80 \pm 2.1$ \\
\hline
\end{tabular}

* Values are expressed as mean $\pm \mathrm{SEM} \%$ and represent the average of 2-3 specimens taken at each time period for control and experimental joints. Differences in values did not reach statistical significance.

+ Includes combined samples of the femoral condyle and tibial plateau cartilage.

changes in proteoglycan metabolism occur in both hind limbs over the time period studied here. However, the water content was $3-4 \%$ higher than that of controls in the knee and patella, but was essentially unchanged in the hip 12-16 weeks postoperatively. Since the $\mathrm{n}$ values for samples at each time period were small, differences in cartilage water content were not found to reach statistical significance. However, the results do show trends of water content in both hind limbs at 4-10 and 12-16 weeks postoperatively.

In vivo ${ }^{35} \mathrm{SO}_{4}{ }^{-2}$ incorporation into proteoglycans. Figure 7 depicts the relative specific activity (dpm/ $/ \mu$ mole uronic acid) from the sum of the total activity found in the associative and dissociative extract fractions of knee (femoral condyles and tibial plateau) cartilage from operated animals compared with unoperated controls. The relative specific activity is plotted against time in weeks following surgery. The mean values are the result of 2-3 determinations at each time point for both experimental and control joints. At each time period, the SEM was $<1,000$ $\mathrm{dpm} / \mu$ mole uronic acid except at 14 weeks, when the values were $\pm 4,070$ for the right knee and $\pm 4,370$ for the left knee. Total dpm/mg wet weight of cartilage was also calculated and showed similar trends in all instances; the results are not reported here.

At 8 weeks postoperatively, the total mean ${ }^{35} \mathrm{SO}_{4}{ }^{-2}$ incorporation in the operated right and contralateral unoperated left side were at or near control values. By 14 weeks, both the right and left knee cartilage showed a sharp increase in relative specific activity. However, by $20-24$ weeks, the relative specific activity in both knees had decreased to levels below the control range. A similar pattern of mean ${ }^{35} \mathrm{SO}_{4}{ }^{-2}$ incorporation was observed in the associative and dissociative cartilage extract fractions when examined individually.

Figure 8 depicts the relative specific activity for the sum of the total activity found in the associative and dissociative extract fractions of femoral head cartilage' from operated animals compared with unoperated controls. Similar to the findings in the knee cartilage, at 8 weeks postoperatively, the total mean ${ }^{35} \mathrm{SO}_{4}{ }^{-2}$ incorporation into cartilage proteoglycans in both hips was below the control range. However, by 12 weeks on the operated right side and 14 weeks on the contralateral unoperated left side, there was a sharp increase in ${ }^{35} \mathrm{SO}_{4}{ }^{-2}$ relative specific activity. As observed in the knee, by 20-24 weeks postoperatively, the values for both femoral heads had decreased to levels below the control range. Mean values at all points had SEM $<4,000 \mathrm{dpm} / \mu$ mole uronic acid except at 14 weeks, when the SEM for the left side was $\pm 8,120$.

In preliminary studies, $71-78 \%$ of the total ${ }^{35} \mathrm{SO}_{4}$-proteoglycans was extracted under associative and dissociative conditions. These values were ob-

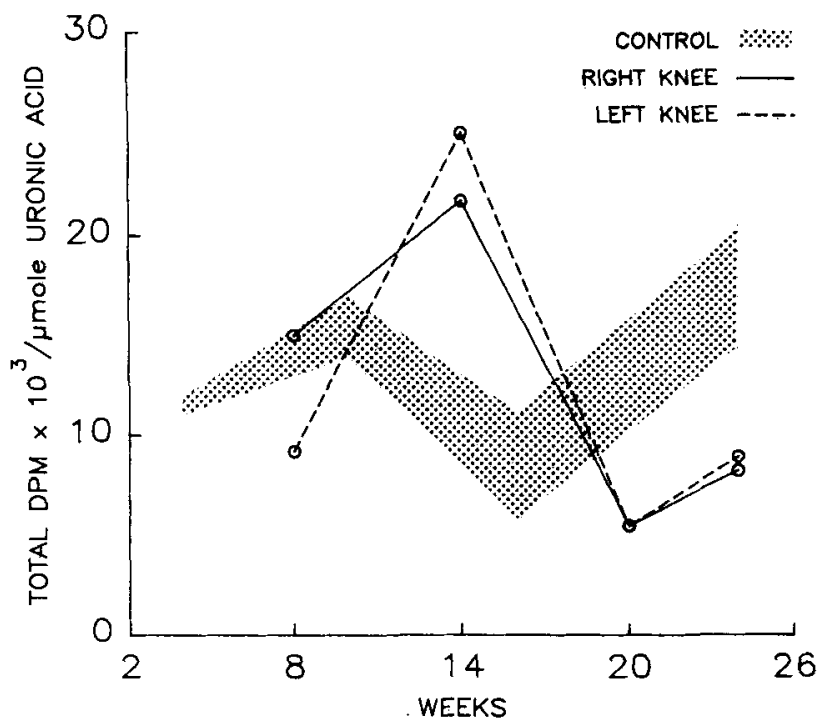

Figure 7. Total disintegrations per minute $\times 10^{3} / \mu$ mole uronic acid (relative specific activity) for the sum of the total activity found in both the associative and dissociative proteoglycan extract fractions of knee (femoral condyles and tibial plateau) cartilage, plotted against time in postoperative weeks. Note the sharp increase in the relative specific activity at $\mathbf{1 4}$ weeks for cartilage from both the right operated knee and left contralateral unoperated knee. 


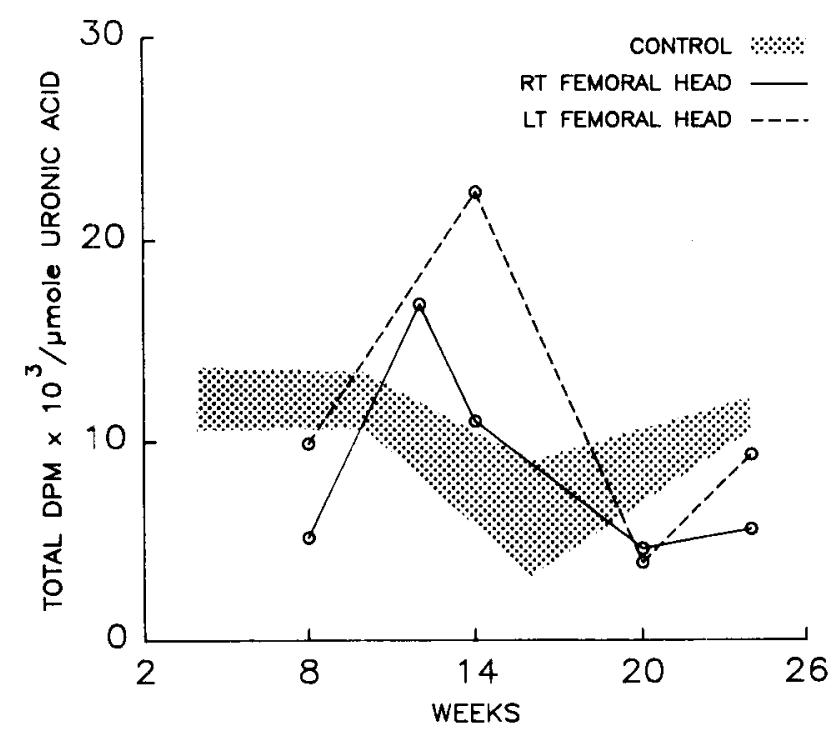

Figure 8. Total disintegrations per minute $\times 10^{3} / \mu$ mole uronic acid (relative specific activity) for the sum of the total activity found in both the associative and dissociative proteoglycan extract fractions of the femoral head, plotted against time in postoperative weeks. Note the sharp increase in the relative specific activity of the right operated femoral head at 12 weeks and the increase in the left contralateral unoperated femoral head at 14 weeks.

tained by adding NCS solubilizer (Amersham Corporation, Arlington Heights, IL) to the cartilage pellet residue and then measuring the total radioactivity in the solubilized pellet by liquid scintillation counting. The percentage of total ${ }^{35} \mathrm{SO}_{4}$-proteoglycans extracted under associative and dissociative conditions was then calculated. These values compared favorably with those previously reported by other investigators $(15,16)$.

Finally, it should be emphasized again that differences in relative specific activity between experimental and control animals were present both in the operated right and unoperated left hind limb joints. This suggests that altered biomechanical forces are acting on each of the individual hind limb joints during the postoperative period under study.

\section{DISCUSSION}

Gross, microscopic, and selected biochemical studies indicate that gluteal muscle myectomy and/or tendotomy in guinea pigs induces changes in articular cartilage compatible with osteoarthritis as early as 10 weeks postoperatively.

Observation of the guinea pigs' gait showed that they had a moderate limp up to 4 weeks after the surgical procedure. The hind limbs were stable through the postoperative period. This altered gait induced abnormal biomechanical stresses, not only on the operated right hind limb, but on the contralateral unoperated left hind limb as well. By gross observation, the unoperated left side appeared to bear most of the loading during the first 4 weeks postoperatively. The guinea pigs also developed a longer stride that at first correlated with their somatic growth, but which later became more apparent when compared with the decrease in stride length seen in the control animals' gaits (Figure 2). This analysis allowed documentation of a gait alteration in the operated animals compared with controls, especially at 16 weeks postoperatively. It is well known that repetitive impulse loading is destructive to cartilage (17). Thus, the postoperative gait abnormality may be the initial factor that triggers the development of the earliest OA changes.

Gross observations of the articular cartilage surfaces in the hip and knee have also shown that the surgical procedure we used induces macroscopic cartilage abnormalities consistent with $\mathrm{OA}$ in both hind limbs. The cartilage developed progressive thinning, roughening, erosions, and pits by 24 weeks postoperatively. The most significant changes were seen in the right operated femoral heads and tibial plateaus, but the left unoperated joints also showed delayed, but progressive, changes. Similar gross abnormalities have been reported in other models of OA including partial meniscectomy in rabbits (18) and section of the anterior cruciate ligament in dogs (3). However, osteophyte formation has been reported to be a very prominent feature in both of these models, whereas it has been a minor feature in the current experimental model.

Histologic evaluation of the joint tissues was also performed. A semiquantitative assessment of the histologic changes was made using a simple $0-3$ scale of values. The parameters included were similar to those described by Mankin and associates wherein a more detailed scale of values was employed to assess osteoarthritic changes (19). In the present model of experimental OA, all joint cartilage showed a loss in metachromatic staining by $20-24$ weeks postoperatively. There was also a progressive loss of matrix integrity in each joint, and the tibial plateaus showed severe fibrillation by 20 weeks postoperatively. A mild increase in cellularity was observed in the femoral heads and tibial plateaus at 16-24 weeks, although actual cloning of cells in the deep layers was rare. In addition, the articular cartilage layer became progres- 
sively thinner in both the femoral heads and tibial plateaus by 24 weeks postoperatively. The most severe histologic abnormalities compatible with $\mathrm{OA}$ were seen in the right operated joints; however, the left unoperated joints also developed similar, less severe changes over the postoperative period of observation ( 24 weeks).

It is important to note that no evidence of an inflammatory cell infiltrate was present in any of the histologic specimens. Also, gross and microscopic observation of the synovial lining layer of the hip and knee revealed no evidence of proliferation or inflammation.

Preliminary biochemical analyses included measurements of articular cartilage water content and determination of in vivo ${ }^{35} \mathrm{SO}_{4}{ }^{-2}$ incorporation into cartilage proteoglycans extractable by both associative and dissociative solvent systems. Change in water content was different in the hip, knee, and patella at 4-10 and 12-16 weeks postoperatively (Table 2). An increase was observed in the operated right hip and knee cartilage at 4-10 weeks which was not sustained in 12-16-week specimens. The patella did not show a significant increase in mean water content at either time period. A moderate increase in water content in articular cartilage from the operated hip and knee is consistent with previous reports by others that this is one of the earliest biochemical changes observed in OA (20-22). It has been suggested that the increase in cartilage water content can be secondary to a breakdown of the fibrillar collagen matrix network or can be caused by a localized decrease in the proteoglycan content in the cartilage matrix (20-22).

An early in vivo increase in ${ }^{35} \mathrm{SO}_{4}{ }^{-2}$ incorporation in cartilage proteoglycans correlated with the gross and histologic findings of moderately progressive signs of osteoarthritis seen at 12-14 weeks. This finding is consistent with the findings in numerous previous reports of a brief period of increased proteoglycan synthesis occurring in the early stages of $\mathrm{OA}$ $(19,22,23)$. As the pathologic changes progressed, a decrease in ${ }^{35} \mathrm{SO}_{4}{ }^{-2}$ proteoglycan labeling was found at 20-24 weeks postoperatively. This change was compatible with a decrease in the rate of proteoglycan synthesis as the osteoarthritic process progressed.

The current experimental model of osteoarthritis showed many of the gross, histologic, and biochemical changes described in other experimental models and in OA in humans $(3-9,24)$. However, most previous experimental models have used an invasive surgical procedure to destabilize a weight-bearing joint and, as a consequence, stimulate a postoperative intraarticular inflammatory response. Under these circumstances, early histopathologic and biochemical events important in the pathogenesis of OA can be obscured or altered by postoperative inflammation. The current OA model does not involve intraarticular surgery; therefore, no inflammatory infiltrate occurs within the joint space at any time. In addition, in the present model, biomechanical forces are applied to the articular cartilages throughout the course of evolution of the OA change.

In addition, in this model, altered biomechanical stress was applied to the operated right hind limb and also to the contralateral unoperated left hind limb. As a consequence, this has produced gross, histologic, and biochemical changes consistent with $\mathrm{OA}$ in all hind limb joints (hip, knee). In the absence of evidence of intraarticular postoperative inflammation, one can conclude that biomechanical factors alone can induce changes in articular chondrocyte function that lead to the development of OA. The degree of biomechanical stress applied to individual joints has led to differences in the rate of OA development in different hind limb joints. The knee developed the most prominent $\mathrm{OA}$ changes and bore the brunt of the biomechanical stress, especially when changes in water content and ${ }^{35} \mathrm{SO}_{4}{ }^{-2}$ incorporation into cartilage proteoglycans are taken into account. Finally, it must be emphasized that unilateral surgical procedures lead to alterations in cartilage integrity and function on both sides of the animal, such that "control" observations cannot be dependably made on contralateral unoperated joints (10). The current model of experimental OA should provide an opportunity to further explore the influences of biomechanics, inflammation, and somatic growth on chondrocyte function and their aggregate impact on the early cellular and biochemical events that lead to osteoarthritis.

\section{ACKNOWLEDGMENTS}

We wish to thank G. William Jourdian, PhD, Professor of Internal Medicine and Biological Chemistry, for his expert advice. We also wish to thank Sharon Miller, BS, for her expert technical support.

\section{REFERENCES}

1. Schwartz ER, Greenwald RA: Experimental models of osteoarthritis. Bull Rheum Dis 30:1030-1033, 1979-80

2. Troyer H: Experimental models of osteoarthritis: a review. Semin Arthritis Rheum 11:362-374, 1982

3. Pond MJ, Nuki G: Experimentally induced osteoarthritis in the dog. Ann Rheum Dis 32:387-388, 1973 
4. Moskowitz RW, Howell DS, Goldberg VM, Muniz O, Pita JC: Cartilage proteoglycan alterations in an experimentally induced model of rabbit osteoarthritis. Arthritis Rheum 22:155-163, 1979

5. Colombo C, Butler M, O'Byrne E, Hickman L, Swartzendruber D, Selwyn M, Steinetz B: A new model of osteoarthritis in rabbits. I. Development of knee joint pathology following lateral meniscectomy and section of the fibular collateral and sesamoid ligaments. Arthritis Rheum 26:875-886, 1983

6. Schwartz ER, Oh WH, Leveille CR: Experimentally induced osteoarthritis in guinea pigs: metabolic responses in articular cartilage to developing pathology. Arthritis Rheum 24:1345-1355, 1981

7. Evans EB, Eggers GWN, Butler JK, Glumel J: Experimental immobilization and remobilization of rat knee joints. J Bone Joint Surg 42A:737-758, 1960

8. Langenskiold A, Michelsson JE, Videman T: Osteoarthritis of the knee in the rabbit produced by immobilization: attempts to achieve a reproducible model for studies on pathogenesis and therapy. Acta Orthop Scand 50:1-14, 1979

9. Troyer $\mathrm{H}$ : The effect of short term immobilization on the rabbit knee joint cartilage: a histochemical study. Clin Orthop 107:249-257, 1975

10. Moskowitz RW, Goldberg VM, Malemud CJ: Metabolic responses of cartilage in experimentally induced osteoarthritis. Ann Rheum Dis 40:584-592, 1981

11. Zannoni V, Lynch $M$, Goldstein S, Sato P: A rapid micromethod for the determination of ascorbic acid in plasma and tissues. Biochem Med 11:41-48, 1974

12. Oegema TR, Hascall VC, Dziewiatkowski DD: Isolation and characterization of proteoglycans from the swarm rat chondrosarcoma. J Biol Chem 250:6151-6159, 1975

13. Schwartz ER, Leveille CR, Stevens JW, Oh WH: Proteoglycan structure and metabolism in normal and osteoarthritic cartilage of guinea pigs. Arthritis Rheum 24:1528-1539, 1981
14. Bitter T, Muir HM: A modified uronic acid carbazole reaction. Anal Biochem 4:330-334, 1962

15. Christner JE, Baker JR, Caterson B: Studies on the properties of the inextractable proteoglycans from bovine nasal cartilage. J Biol Chem 258:14335-14341, 1983

16. Sajdera SW, Hascall VC: Proteinpolysaccharide complex from bovine nasal cartilage: a comparison of low and high shear extraction procedures. J Biol Chem 244:77-87, 1969

17. Radin EL: Mechanical aspects of osteoarthrosis. Bull Rheum Dis 26:862-865, 1975-76

18. Moskowitz RW, Davis W, Sammarco J, Martens M, Baker J, Mayor M, Burstein AH, Frankel VH: Experimentally induced degenerative joint lesions following partial meniscectomy in the rabbit. Arthritis Rheum $16: 397-405,1973$

19. Mankin HJ, Dorfman H, Lippiello L, Zarins A: Biochemical and metabolic abnormalities in articular cartilage from osteoarthritic human hips. II. Correlation of morphology with biochemical and metabolic data. J Bone Joint Surg 53A:523-537, 1971

20. Muir H, Maroudas A, Wingham J: The correlation of fixed negative charge with glycosaminoglycan content of human articular cartilage. Biochem Biophys Acta 177:492-500, 1969

21. Muir H: Cartilage structure and metabolism and basic changes in degenerative disease. Aust NZ J Med 8:1-5, 1978

22. Mankin HJ, Lippiello L: Biochemical and metabolic abnormalities in articular cartilage from osteoarthritic human hips. J Bone Joint Surg 52A:424-433, 1970

23. Sandy JD, Adams ME, Billingham MEJ, Plaas A, Muir $\mathrm{H}$ : In vivo and in vitro stimulation of chondrocyte biosynthetic activity in early experimental osteoarthritis. Arthritis Rheum 27:388-397, 1984

24. Ryu J, Treadwell BV, Mankin HJ: Biochemical and metabolic abnormalities in normal and osteoarthritic human articular cartilage. Arthritis Rheum 27:49-57, 1984 